\title{
Bilateral sympathectomy improves postinfarction left ventricular remodeling and function
}

\author{
Fernando Luiz Zanoni, DVM, PhD, Rafael Simas, PhD, Raphael Grillo da Silva, DVM, \\ Ana Cristina Breithaupt-Faloppa, PhD, Raphael dos Santos Coutinho e Silva, BSc, \\ Fábio Biscegli Jatene, MD, PhD, and Luiz Felipe P. Moreira, MD, PhD
}

\section{ABSTRACT}

Objectives: To evaluate the influence of bilateral or left sympathectomy on left ventricular remodeling and function after myocardial infarction in rats.

Methods: Myocardial infarction was induced in rats by ligation of the left anterior descending coronary. Seven days later, rats were divided into 4 groups: the myocardial infarction, myocardial infarction with left sympathectomy, myocardial infarction with bilateral sympathectomy, and sham groups. After 8 weeks, left ventricular function was evaluated with the use of a pressure-volume conductance catheter under steady-state conditions and pharmacological stress. Infarct size and extracellular matrix fibrosis were evaluated, and cardiac matrix metalloproteinases and myocardial inflammatory markers were analyzed.

Results: The myocardial infarction and left sympathectomy group had an increased end diastolic volume, whereas the bilateral sympathectomy group had a mean end-diastolic volume similar to that of the sham group $(P<.002)$. Significant reduction in ejection fraction was observed in the myocardial infarction and left sympathectomy group, whereas it was preserved after bilateral sympathectomy $(P<.001)$. In response to dobutamine, left ventricular contractility increased in sham rats, rising stroke work, cardiac output, systolic volume, end-diastolic volume, ejection fraction, and $\mathrm{dP} / \mathrm{dt}$ max. Only bilateral sympathectomy rats had significant increases in ejection fraction $(P<.001)$ with dobutamine. Fibrotic tissue and matrix metalloproteinase expression decreased in the bilateral sympathectomy group compared to that in the myocardial infarction group $(P<.001)$ and was associated with left ventricular wall thickness maintenance and better apoptotic markers in noninfarcted myocardium.

Conclusions: Bilateral sympathectomy effectively attenuated left ventricular remodeling and preserved systolic function after myocardial infarction induction in rats. (J Thorac Cardiovasc Surg 2017;153:855-63)

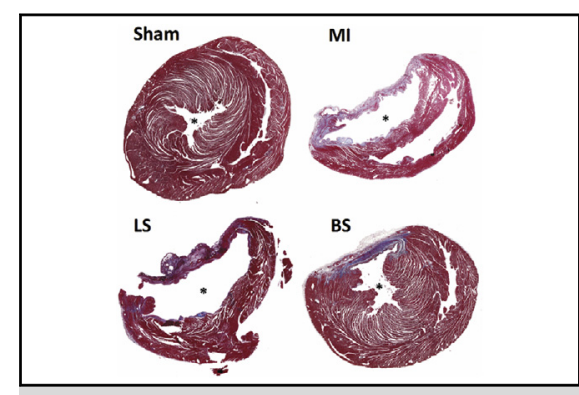

Left ventricle fibrosis is reduced by bilateral sympathectomy.

\section{Central Message}

Bilateral sympathectomy attenuates left ventricular remodeling and preserves ventricular function after myocardial infarction.

\section{Perspective}

We hypothesized that bilateral sympathectomy may positively influence ventricular remodeling and clinical evolution in compromised left ventricular function after myocardial infarction. In rats, sympathectomy decreases substitution of the normal extracellular matrix in the fibrotic tissue by regulation of metalloproteinases and apoptosis.

See Editorial Commentary page 864.
Despite continuous therapeutic advances, the mortality and morbidity of chronic heart failure continue to increase, and new treatment options are needed. Ventricular remodeling

\footnotetext{
From the Laboratory of Cardiovascular Surgery and Pathophysiology of Circulation, Heart Institute (Incor), Department of Cardiopneumology, Sao Paulo University Medical School, Sao Paulo, Brazil.

Supported by FAPESP grant \#2013/06130-3. The design of the study, collection and analysis of data, and writing and publication of the manuscript were performed independently by the authors, without influence from the sponsor.

Received for publication June 28, 2016; revisions received Oct 28, 2016; accepted for publication Nov 14, 2016; available ahead of print Dec 18, 2016

Address for reprints: Fernando Luiz Zanoni, DVM, PhD, Laboratory of Cardiovascular Surgery and Circulation Pathophysiology, Heart Institute (Incor),

Sao Paulo University Medical School, Sao Paulo, Brazil, Av. Dr Eneas Carvalho de Aguiar, 44, Sao Paulo, SP 05403-900, Brazil (E-mail: zanoni@usp.br).

$0022-5223 / \$ 36.00$

Copyright (C) 2016 by The American Association for Thoracic Surgery

http://dx.doi.org/10.1016/j.jtcvs.2016.11.037
}

has been associated with poor long-term outcomes after myocardial infarction (MI), and the progression of ischemic cardiomyopathy is characterized by several factors, including augmented sympathetic activity.

New nonpharmacologic treatments of heart failure have focused on increasing parasympathetic activity via the use of different stimulation approaches or sympathetic blockade with renal sympathetic denervation and left cardiac sympathectomy. ${ }^{1,2}$ The preclinical results of these

Scanning this QR code will take you to a video and supplemental table for the article.

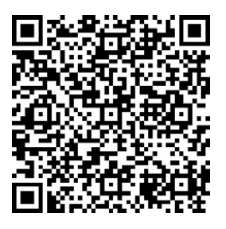




$$
\begin{aligned}
& \text { Abbreviations and Acronyms } \\
& \begin{aligned}
\text { BS } & =\text { bilateral sympathectomy } \\
\text { BNP } & =\text { brain natriuretic peptide } \\
\text { CO } & =\text { cardiac output } \\
\text { IL } & =\text { interleukin } \\
\text { LAD } & =\text { left anterior descending } \\
\text { LS } & =\text { left sympathectomy } \\
\text { LV } & =\text { left ventricular } \\
\text { LVEDV } & =\text { left ventricular end-diastolic volume } \\
\text { LVEF } & =\text { left ventricular ejection fraction } \\
\text { LVSW } & =\text { left ventricle stroke work } \\
\text { MI } & =\text { myocardial infarction } \\
\text { MMP } & =\text { matrix metalloproteinase } \\
\text { PCR } & =\text { polymerase chain reaction } \\
\text { PBS } & =\text { phosphate-buffered saline } \\
\text { SG } & =\text { stellate ganglion } \\
\text { SV } & =\text { stroke volume } \\
\text { VEGF } & =\text { vascular endothelial growth factor }
\end{aligned}
\end{aligned}
$$

procedures are encouraging, but initial clinical trials have been unpredictable and harmful. ${ }^{1-3}$ A preliminary study reported modest efficacy of left cardiac sympathetic denervation in patients with dilated cardiomyopathy, ${ }^{4}$ and this procedure has been shown to modify favorably the incidence of life-threatening arrhythmia. ${ }^{5}$ Besides the decrease of sympathetic activity, several mechanisms of neuromodulation in these new interventional modalities probably contribute to their favorable effect, including reduced apoptosis, metalloproteinase inhibition, and augmentation of the anti-inflammatory effect of vagal activity.

In this study, we evaluated the influence of bilateral or left thoracic sympathectomy on left ventricular (LV) remodeling and function after MI induction in rats. The possible involvement of alterations of the cardiac extracellular matrix and myocardial inflammatory compromise during ventricular remodeling also was investigated.

\section{MATERIALS AND METHODS}

All experiments were performed under the ethical principles for animal research adopted by the Brazilian College of Animal Experimentation. Approval of the Animal Subject Committee of the University of Sao Paulo Medical School was obtained. This work was supported by FAPESP grant (\#2013/06130-3) and received ethics committee approval (CEP-FMUSP \#145/12). A demonstration of some experimental procedures are briefly shown in Video 1.

\section{Anesthesia, Analgesia, and MI Induction}

Male Wistar rats weighing 300 to $350 \mathrm{~g}$ were anesthetized with isoflurane in $\mathrm{O}_{2}\left(\mathrm{FiO}_{2} 100 \%\right)$. After induction of anesthesia with $5 \%$ isoflurane and orotracheal intubation, the animals were ventilated mechanically in a rodent ventilator (Harvard Apparatus, Holliston, Mass) with tidal volume of $7 \mathrm{~mL} / \mathrm{kg}, 70$ breaths per minute.

After trichotomy and antisepsis, left thoracotomy was performed, and MI was induced by ligation of the left anterior descending artery (LAD) $(\mathrm{n}=77)$, as described previously. ${ }^{8}$ Sham rats $(\mathrm{n}=14)$ were submitted only to thoracotomy.

\section{Chemical Sympathectomy and Experimental Groups}

Seven days after MI induction, rats were divided randomly in 3 groups: MI only, left sympathectomy (LS) with MI, and bilateral sympathectomy (BS) with MI. Sympathectomy was performed by chemical sclerosis of stellate ganglion (SG) with $200 \mu \mathrm{L}$ of absolute ethanol. To access the $\mathrm{SG}$, we used the percutaneous lateral technique, as described by Gulcu and colleagues. ${ }^{9}$ To summarize in brief, rats were placed in the left or right lateral decubitus position, and after palpation of the $\mathrm{C} 7$ vertebra, the lower part was fixed. An insulin injector was inserted laterally towards the vertebral surface, and ethanol was injected when the needle touched this surface. To promote LS, ethanol was injected on the left side; to promote BS, ethanol was injected on both sides of the C7 vertebra. Sympathectomy by SG sclerosis was confirmed through observation of ipsilateral ptosis.

\section{Function Evaluation}

Eight weeks after MI induction, rats were anesthetized as described previously. A 2F microtip pressure-conductance catheter (SPR-838; Millar Instruments, Houston, Tex) was inserted into the right carotid artery and advanced into the left ventricle. Ten minutes after stabilization, signals were recorded with a pressure-volume conductance System (MPVS-Ultra, Millar Instruments, Houston, Tex) connected to a data acquisition system (PowerLab, AD Instruments, Colorado Springs, Colo). LV stroke work (LVSW), stroke volume (SV), cardiac output (CO), left ventricular end-diastolic volume (LVEDV), LV end-systolic volume, left ventricular end-systolic pressure, left ventricular ejection fraction (LVEF), heart rate, maximal slope of the $\mathrm{LV}$ systolic pressure increment ( $\mathrm{dP} / \mathrm{dt} \max )$, and time constant of LV pressure decay (tau) were obtained at steadystate conditions and after pharmacologic stress. The dobutamine protocol was performed via a continuous infusion of $2.5 \mu \mathrm{g} / \mathrm{kg} / \mathrm{min}$ for 10 minutes, followed by 5 minutes of data acquisition. Thereafter, the dosage was increased to $5.0 \mu \mathrm{g} / \mathrm{kg} / \mathrm{min}$ for 10 minutes, followed by 5 minutes of data acquisition. In addition to the general parameters obtained at steady-state and after pharmacologic stress, myocardial mechanical efficiency was estimated via the ratio between LVSW and pressurevolume area under the different conditions. ${ }^{10}$

We injected $50 \mu \mathrm{L}$ of $7.5 \%$ hypertonic saline at the end of each experiment, and parallel conductance volume was calculated and used for correction of the cardiac mass volume. Volume calibration was performed with fresh heparinized warm blood.

\section{Histological Analysis}

At the end of the experiments, rats were euthanized by exsanguination. The hearts were arrested in diastole by infusion of hyperkalemic solution

\section{Myocardial Infarction}

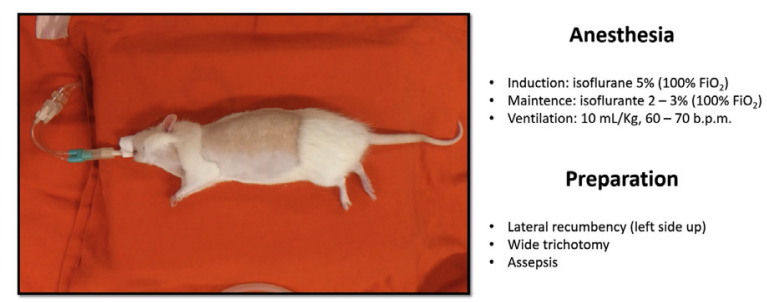

VIDEO 1. A short demonstration of the rat model of myocardial infarction and stellate ganglion chemical sympathectomy. Video available at: http:// www.jtcvsonline.org/article/S0022-5223(16)31632-4/addons. 
$(19 \% \mathrm{KCl})$ and immersed in buffered formalin for 24 hours. Median slices at the level of the papillary muscles were dehydrated, embedded in paraffin, cut into a series of 3- $\mu \mathrm{m}$-thick slices, and stained with hematoxylin-eosin and Masson's trichrome. The thickness of the LV, right ventricle, and interventricular septum, as well the quantification of the scar area (infarct size) were performed. Using the stained myocardial cuts, we calculated the percentage of connective tissue in the LV free wall by determining the area under $20 \times$ augmentation.

\section{Heart Homogenate and Enzyme-Linked Immunosorbent Assays}

Heart homogenates were prepared via the quantification of enzymelinked immunosorbent assay of some substances. A part of the noninfarcted LV wall was weighed and homogenized with phosphate-buffered saline (PBS; $1 \mathrm{~mL} / 100 \mathrm{mg}$ tissue) in a TissueLyser. These homogenates were centrifuged at $3000 \mathrm{~g}$ for 5 minutes at $4^{\circ} \mathrm{C}$, and thereafter the supernatants were centrifuged again at $5000 \mathrm{~g}$ for 20 minutes at $4^{\circ} \mathrm{C}$. Aliquots of the $\mathrm{LV}$ homogenate supernatant were stored at $-80^{\circ} \mathrm{C}$.

The concentration of interleukin (IL)-6 and IL-10 (R\&D Systems Inc, Minneapolis, Minn), catecholamines (adrenaline and noradrenaline; MyBioSource, San Diego, Calif), brain natriuretic peptide (BNP)-32 (Abcam, Cambridge, $\mathrm{UK}$ ), and vascular endothelial growth factor (VEGF; R\&D Systems Inc) were determined with the use of commercially available kits, according to the manufacturer's instructions. The results were expressed as $\mathrm{ng} / \mathrm{g}$ or $\mathrm{pg} / \mathrm{g}$ tissue.

\section{Cardiac Matrix Metalloproteinases (MMPs) and VEGF mRNA Analysis}

Immediately after euthanasia, noninfarcted LV myocardial samples were collected, quickly frozen in liquid nitrogen, and stored at $-80^{\circ} \mathrm{C}$. They were homogenized in lysis buffer, and the RNA was isolated via the use of an mirVana miRNA Isolation Kit with phenol (Ambion, Carlsbad, Calif) and quantified by measuring optical density at $260 \mathrm{~nm}$. A $260 / 280 \mathrm{~nm}$ optical density ratio of $\sim 2.0$ ensured the purity of the RNA. Reverse transcription reaction was completed with the HighCapacity cDNA Reverse Transcription Kit (Applied Biosystems, Foster City, Calif). Quantitative real-time polymerase chain reaction (PCR) was performed in duplicates of each sample in a volume of $10 \mu \mathrm{L}$ in each well containing cDNA $(1 \mu \mathrm{L})$, TaqMan Universal PCR MasterMix $(5 \mu \mathrm{L})$, and a TaqMan Gene Expression Assay $(0.5 \mu \mathrm{L})$ with the StepOnePlus Real-Time PCR System (Applied Biosystems) for the following targets: MMP-2 (assay ID: Rn01538170_m1), MMP-9 (assay ID: Rn00579162_m1), and VEGF (assay ID: Rn01511601_m1). Data of gene expression were normalized to a reference gene (glyceraldehyde-3phosphate dehydrogenase; assay ID: Rn01775763_g1). Results were expressed as values normalized in relation to a positive calibrator obtained with a pool of cDNAs from the overall data of the sham group.

\section{Caspase 3 and Bcl-2 Apoptotic Markers}

Serial 4-mm sections of hearts embedded paraffin were deparaffinized and hydrated. The sections were incubated with $3 \% \mathrm{H}_{2} \mathrm{O}_{2}$ and Pierce solution (ThermoFisher Scientific, Waltham, Mass) to block endogenous peroxidase and biotin, respectively. For immunodetection of BCL-2 and caspase-3, tissue sections were incubated overnight at $4{ }^{\circ} \mathrm{C}$ with rat monoclonal antibody anti-Caspase- 3 and anti-BCL-2 (Abcam, Cambridge, Mass), diluted 1:100 in Tris-buffered saline with Tween 20 with bovine serum albumin $1 \%$. After the slides were washed with PBS, sections were incubated with streptavidin (R\&D Systems Inc) that was diluted 1:500 in PBS for 1 hour at room temperature, developed with 3.3'-diaminobenzidine (Sigma Chemical Co, St Louis, Mo), and counterstained with hematoxylin. Analyses were performed in noninfarcted LV tissue with Image-Pro Plus, version 4.1 (Media Cybernetics, Silver Spring, Md). Results are presented as mean optical density.

\section{Statistical Analysis}

All results were expressed as means \pm standard deviation, and statistical analysis was performed with commercially available software (Prism 6.0, GraphPad Software Inc, La Jolla, Calif). Differences between groups were assessed by the use of 1-way analysis of variance or 2-way analysis of variance with repeated measures over the condition factor in the LV function evaluation. These tests were followed by pair-wise comparisons between sham or sympathectomy groups in relation to MI group with Bonferroni corrections with $P$ values adjusted to account for multiple comparisons. Kruskal-Wallis test, followed by Dunn's multiple comparison test, was used to assess the differences between sham or sympathectomy groups in relation to MI group, when data were not distributed normally. $P$ values less than .05 were considered statistically significant.

\section{RESULTS}

Ninety-one rats were used: 77 were submitted to MI, of which $35(45 \%)$ died. Most deaths (25 rats) occurred in the first 24 hours post-MI as the result of arrhythmia, and the remaining 10 deaths occurred up to 8 weeks after induction, but the mortality rate did not differ among groups. During the 8 -week protocol, body weight gain was significantly lower $(P<.05)$ in MI $(158 \pm 49 \mathrm{~g})$ and $\mathrm{LS}(156 \pm 33 \mathrm{~g})$ rats than in sham rats $(195 \pm 38 \mathrm{~g})$. The body weight gain was not significantly different in the BS $(169 \pm 31 \mathrm{~g})$ and sham groups.

\section{Infarct Size and Ventricular Remodeling}

The ligation of LAD induced successfully a mean infarct size of $37 \%$ in the MI group (Table 1). BS significantly attenuated the extent of the infarct area, a situation that was not observed in the LS group. Similar results were observed when we compared wall thickness. Compared with that in the sham group, significant reductions in the septal and LV wall thickness were observed in the MI and LS groups, whereas no difference was observed in the BS group. The thickness of the right ventricle did not differ between groups.

Microscopic evaluation of the LV myocardium stained with Masson trichrome revealed substantial connective tissue in the LV free wall in the MI and LS groups compared with that in the sham group, whereas a less-important compromise of the extracellular matrix was documented in rats in the BS group (Figure 1). The percentages of connective tissue in the LV free wall confirm the significant decrease in fibrosis in BS rats, compared with that in MI rats (Table 1).

\section{Functional Analysis}

Steady-state hemodynamics. The data presented in Table 2 show that under steady-state conditions, LVEF decreased significantly in the MI and LS groups 8 weeks after LAD ligation compared with the sham group. In parallel, compared with sham rats, MI and LS rats exhibited a trend to an increase in LVEDV. Both LVEF and LVEDV were preserved in the BS group. The other hemodynamic 
TABLE 1. Heart morphometric and histologic data

\begin{tabular}{lccccc}
\hline \multicolumn{1}{c}{ Groups } & Infarct size, \% & LV fibrosis, \% & RV wall thickness, mm & Septal thickness, mm & LV wall thickness, mm \\
\hline Sham, $\mathrm{n}=7$ & - & $9.3 \pm 10.9$ & $1.22 \pm 0.13$ & $2.79 \pm 0.15$ & $3.62 \pm 0.15$ \\
MI, $\mathrm{n}=7$ & $36.5 \pm 8.8$ & $73.6 \pm 21.1^{*}$ & $1.20 \pm 0.38$ & $1.91 \pm 0.56 \dagger$ & $1.45 \pm 0.97 *$ \\
LS, $\mathrm{n}=7$ & $31.1 \pm 10.6$ & $68.4 \pm 15.6^{*}$ & $0.97 \pm 0.23$ & $1.89 \pm 0.63 \dagger$ & $2.40 \pm 0.67 \dagger$ \\
BS, $\mathrm{n}=7$ & $21.5 \pm 6.9 \ddagger$ & $22.4 \pm 8.5 \dagger \S$ & $1.06 \pm 0.20$ & $2.39 \pm 0.65$ & $2.85 \pm 0.52 \S$ \\
\hline
\end{tabular}

Values are expressed as mean \pm standard deviation for 7 animals per group. $L V$, Left ventricle; $R V$, right ventricular; $M I$, myocardial infarction; $L S$, left sympathectomy; $B S$, bilateral sympathectomy. ${ }^{*} P<.001$ versus sham. $\dagger P<.05$ versus sham. $\ddagger P=.01$ versus MI. $\S P<.001$ versus MI

measurements (LVSW, CO, SV, LV end-systolic volume, LVEDP, left ventricular end-systolic pressure, HR, dP/dt max, and tau) did not show significant changes between groups under steady-state conditions.

Hemodynamics during DBT stimulation. During DBT infusion, global cardiac function increased in shamoperated rats, evidenced by greater LVSW, CO, SV, LVEDV, HR, LVEF, and $\mathrm{dP} / \mathrm{dt}$ max. In the 3 infarcted groups (MI, LS, and BS), only slight modifications of these variables were observed (Table 2); however, systolic function was preserved in BS rats, which exhibited a significant increase in LVEF $(P<.01)$ after DBT $5.0 \mu \mathrm{g} / \mathrm{kg} / \mathrm{min}$, compared with MI rats. Figure 2 also shows that the overall efficiency of contractile activity was significantly better in the BS and sham groups than in the MI group in steady-state conditions and under DBT infusion. The other parameters did not show significant differences between groups after DBT infusion.

\section{Myocardial Cytokines, BNP-32, and Catecholamines}

No differences were observed in myocardial IL-6 concentration between groups, but compared with sham, IL-10 concentration decreased significantly in the LS and
BS groups $(P<.01)$. A slight but not significant increase in BNP-32 concentration was observed only in the MI group. No differences were observed in adrenaline concentrations in myocardial homogenate between groups, whereas a marginally significant noradrenaline concentration increase was documented in the MI group compared with sham $(P=.04)$ (Table E1).

\section{Extracellular Matrix Regulation Markers}

Myocardial gene expression of MMP-2 and MMP-9. Relative to the sham group, MMP-2 expression in LV did not change in the MI group 8 weeks after LAD ligation. Sympathectomy reduced MMP-2 expression 10.6-fold in the BS group $(P<.001)$ compared with MI. MMP-9 expression was increased 2.3-fold in MI and 1.7-fold in LS, relative to sham. The BS group showed great reduction in MMP expression, 20-fold lower than that in the MI group (Figure 3).

VEGF gene expression and protein content. No significant increases in VEGF gene expression or protein content were observed in MI rats compared with sham. In contrast, VEGF gene expression was reduced in the BS group without statistical significance but showed a great decrease

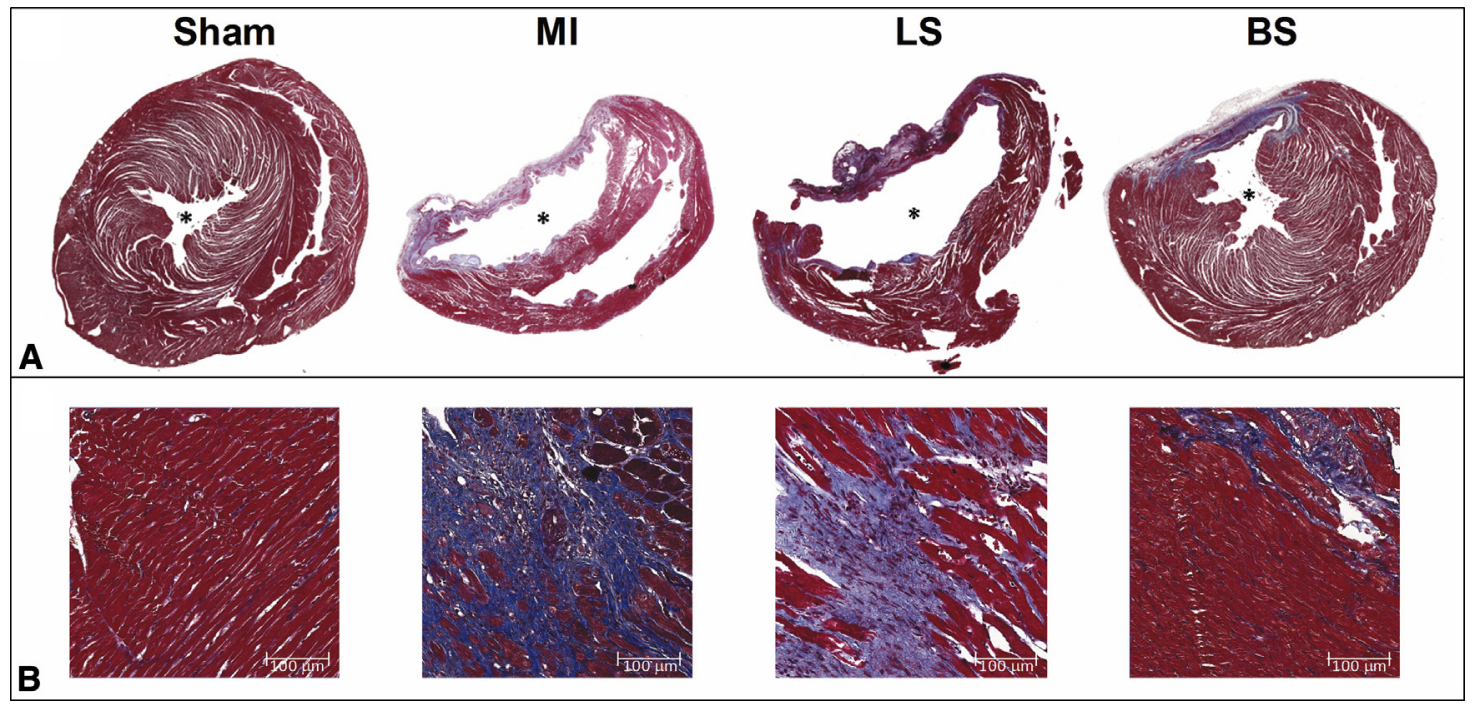

FIGURE 1. Representative photomicrographs of the left ventricular myocardium stained with Masson's trichrome in gross section (A) or with $20 \times$ augmentation (B), showing the intensity of fibrotic tissue in the 3 groups. *Represents the lumen of the left ventricle. MI, Myocardial infarction; $L S$, left sympathectomy; $B S$, bilateral sympathectomy. 
TABLE 2. LV hemodynamics parameters obtained by P-V loop analysis

\begin{tabular}{|c|c|c|c|c|}
\hline & Sham, $n=7$ & $\mathrm{MI}, \mathrm{n}=7$ & LS, $\mathbf{n}=7$ & BS, $\mathbf{n}=7$ \\
\hline \multicolumn{5}{|c|}{ Stroke work, $\mathrm{mm} \mathrm{Hg}^{*} \mu \mathrm{L}$} \\
\hline Steady-state & $6671 \pm 2146$ & $5950 \pm 3235$ & $7857 \pm 4649$ & $6771 \pm 2129$ \\
\hline DBT 2.5 & $11,509 \pm 9223$ & $8085 \pm 4578$ & $7509 \pm 4324$ & $9124 \pm 3373$ \\
\hline DBT 5.0 & $12,150 \pm 13,844$ & $7871 \pm 4335$ & $7211 \pm 4598$ & $8048 \pm 3071$ \\
\hline \multicolumn{5}{|c|}{ Stroke volume, $\mu \mathrm{L}$} \\
\hline Steady-state & $79 \pm 15$ & $67 \pm 23$ & $73 \pm 38$ & $69 \pm 19$ \\
\hline DBT 2.5 & $120 \pm 75$ & $84 \pm 30$ & $77 \pm 29$ & $86 \pm 27$ \\
\hline DBT 5.0 & $130 \pm 110$ & $83 \pm 30$ & $85 \pm 36$ & $88 \pm 28$ \\
\hline \multicolumn{5}{|c|}{ End-diastolic volume, $\mu \mathrm{L}$} \\
\hline Steady-state & $133 \pm 26$ & $214 \pm 41$ & $248 \pm 103$ & $133 \pm 78$ \\
\hline DBT 2.5 & $218 \pm 186$ & $204 \pm 59$ & $238 \pm 77$ & $143 \pm 81$ \\
\hline DBT 5.0 & $203 \pm 182$ & $205 \pm 76$ & $248 \pm 58$ & $135 \pm 66$ \\
\hline \multicolumn{5}{|c|}{ Ejection fraction, $\%$} \\
\hline Steady-state & $62 \pm 8$ & $33 \pm 14^{*}$ & $35 \pm 17 *$ & $51 \pm 14$ \\
\hline DBT 2.5 & $64 \pm 13$ & $46 \pm 23$ & $38 \pm 20^{*}$ & $66 \pm 19$ \\
\hline DBT 5.0 & $71 \pm 11$ & $48 \pm 27$ & $38 \pm 19 *$ & $77 \pm 21 \dagger$ \\
\hline \multicolumn{5}{|c|}{$\mathrm{dP} / \mathrm{dt} \max , \mathrm{mm} \mathrm{Hg} / \mathrm{s}$} \\
\hline Steady-state & $5121 \pm 1208$ & $5016 \pm 2009$ & $5865 \pm 2345$ & $4594 \pm 957$ \\
\hline DBT 2.5 & $6162 \pm 1894$ & $6309 \pm 2663$ & $7420 \pm 3158$ & $5959 \pm 934$ \\
\hline DBT 5.0 & $6238 \pm 2448$ & $6456 \pm 2759$ & $7644 \pm 3490$ & $5965 \pm 1234$ \\
\hline \multicolumn{5}{|l|}{ Tau, ms } \\
\hline Steady-state & $10.33 \pm 1.92$ & $13.95 \pm 10.80$ & $15.47 \pm 12.86$ & $11.50 \pm 3.37$ \\
\hline DBT 2.5 & $9.27 \pm 3.54$ & $11.56 \pm 10.15$ & $12.37 \pm 10.67$ & $9.07 \pm 2.09$ \\
\hline DBT 5.0 & $8.78 \pm 3.07$ & $10.98 \pm 10.00$ & $11.95 \pm 9.94$ & $8.65 \pm 1.73$ \\
\hline
\end{tabular}

Data are presented as mean \pm standard deviation for 7 animals in each group. Data not shown for cardiac output, end-diastolic volume, end-diastolic pressure, end-systolic pressure, and heart rate. $L V$, Left ventricle; $P-V$, pressure volume; $M I$, myocardial infarction; $L S$, left sympathectomy; $B S$, bilateral sympathectomy; $D B T 2.5$, data acquired with dobutamine $2.5 \mu \mathrm{g} / \mathrm{kg} / \mathrm{min} ; D B T 5.0$, data acquired with dobutamine $5.0 \mu \mathrm{g} / \mathrm{kg} / \mathrm{min}$. $* P<.05$ versus sham. $\dagger P=.01$ versus MI.

of its protein content in the LV myocardium in relation to that in MI rats (Figure 4).

\section{Myocardial Apoptosis}

MI and LS rats exhibited a significantly decreased expression of BCL-2, an antiapoptotic protein, compared

\section{Myocardial Efficiency}

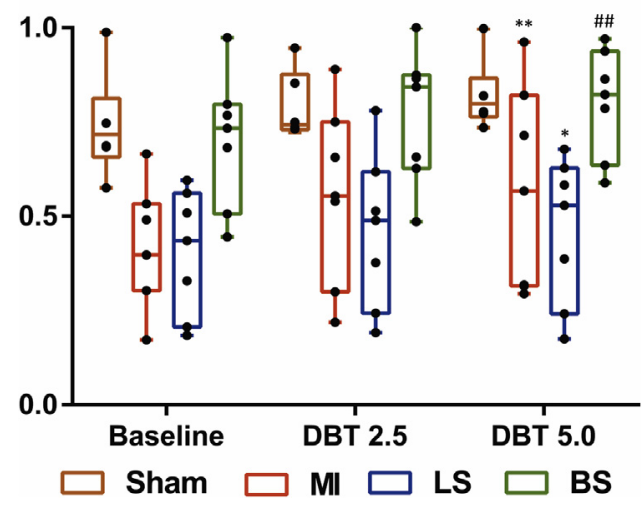

FIGURE 2. Myocardial efficiency estimated by P-V loop analysis at steady-state and under DBT infusion at 2.5 and $5.0 \mu \mathrm{g} / \mathrm{kg} / \mathrm{min}$. Data are presented as mean \pm standard deviation for 7 animals in each group. $D B T$, Dobutamine; $M I$, myocardial infarction; $L S$, left sympathectomy; $B S$, bilateral sympathectomy. $* P<.005$ versus sham; $* * P<.05$ versus sham; ${ }^{\# \#} P<.05$ versus MI. with sham rats, whereas this protein expression was preserved in the BS group. At this time, the expression of caspase-3, a proapoptotic protein, was significantly increased in MI and LS, whereas BS exhibited values comparable to those of sham rats (Figure 5).

\section{DISCUSSION}

We evaluated the effects of permanent sympathetic block at the level of the SG on postinfarction remodeling and ventricular function in rats. Over 8 weeks after MI induction, bilateral sympathetic blockade attenuated ventricular remodeling with the maintenance of LV diastolic volume and wall thickness, thereby preserving ventricular function. Attenuation of LV structural and functional deterioration in BS was associated with decreased apoptotic activity and reduced expression of metalloproteinases and VEGF in the cardiac tissue.

Although pharmacologic therapy effectively controls ventricular remodeling, thus delaying heart failure, the search for new treatment strategies is ongoing. In recent years, inhibition of sympathetic activity by denervation has been evaluated. In a study with infarcted rats, renal denervation improved hemodynamics and reduced neurohumoral activation and ventricular remodeling. ${ }^{11}$ Down-regulation of sympathetic activity through vagal stimulation also has been evaluated, and there was less 
MMP-2

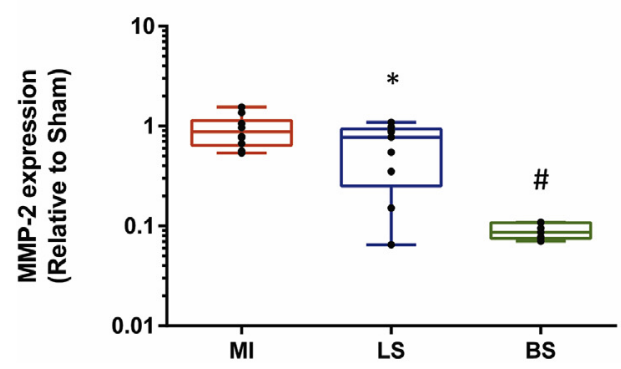

MMP-9

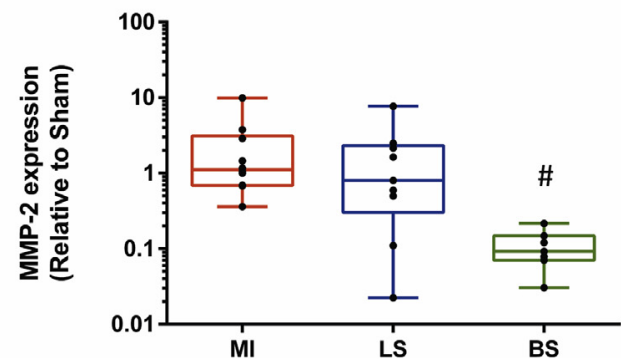

FIGURE 3. Gene expression of MMP-2 and -9 in left ventricular myocardium. Data are presented as mean \pm standard deviation for 7 animals in each group. ${ }^{*} P<.05$ versus MI; ${ }^{\#} P<.001$ versus MI. $M M P-2$, Metalloproteinase-2; MI, myocardial infarction; $L S$, left sympathectomy; $B S$, bilateral sympathectomy.

ventricular remodeling and better LV contractility with vagus nerve stimulation for 72 hours 3 weeks after the onset of MI in rats. ${ }^{12}$

In humans, sympathetic blockade by thoracic epidural anesthesia or thoracic sympathectomy has also been investigated. Guo and colleagues ${ }^{3}$ investigated the effects of sympathetic epidural blockade in dilated cardiomyopathy and observed increased LVEF and a reduction in heart chamber size in patients who underwent this procedure. Sympathetic cardiac denervation in patients with left heart failure proved to be safe, and our group also documented modest changes in LV function and quality of life with this procedure for dilated cardiomyopathy treatment. ${ }^{5}$ In this regard, despite the possible advantages related to left cardiac sympathetic denervation, as the maintenance of myocardial performance during exercise or the absence of post-denervation hypersensitivity, ${ }^{6}$ the relevance of unilateral or BS for heart failure control remains unclear.

Furthermore, inhibition of myocardial extracellular matrix progression by metalloproteinases blockade has been described as another important objective. ${ }^{13}$ Both mechanisms are involved in the myocardial remodeling process in dilated and ischemic cardiomyopathies. ${ }^{14,15}$ Sympathetic hyperactivity has profound effects in the long term on rough function and cardiac structure, and also is associated with changes in the myocardial extracellular matrix. ${ }^{15,16}$ A recent study demonstrated that sympathetic hyperactivity seems to be a cause of myocardial extracellular matrix disruption, directly contributing to the development of cardiac dysfunction. ${ }^{17}$

In our study, ligation of the LAD coronary induced moderate $\mathrm{MI}$ in rats, progressing to ventricular remodeling within 8 weeks. The substantial mortality observed in the first 24 hours post-MI is well reported and is due particularly to severe ventricular arrhythmia. ${ }^{18}$ An absence of significant differences between the studied groups was observed regarding catecholamines, cytokines, and BNP concentration at the myocardial tissue in the long term after MI, despite the significant impact of bilateral sympathetic denervation on LV remodeling and function. The increase in noradrenalin concentration in LV tissue observed in the MI group indicates a high sympathetic activity sustained by the progression of MI. In this regard, these variables are important for the development of ischemic cardiomyopathy, especially when ischemic injury and postinfarction inflammation occur. ${ }^{13}$ After the onset of myocardial necrosis, an inflammatory reaction, orchestrated by inflammatory cells (neutrophils, macrophages, dendritic cells, and lymphocytes), cytokines (IL-6 and IL-10), and MMPs (MMP-2 and MMP-9) will lead to scar formation, angiogenesis, and infarct expansion. This infarct expansion may induce a ventricular aneurysm, and the
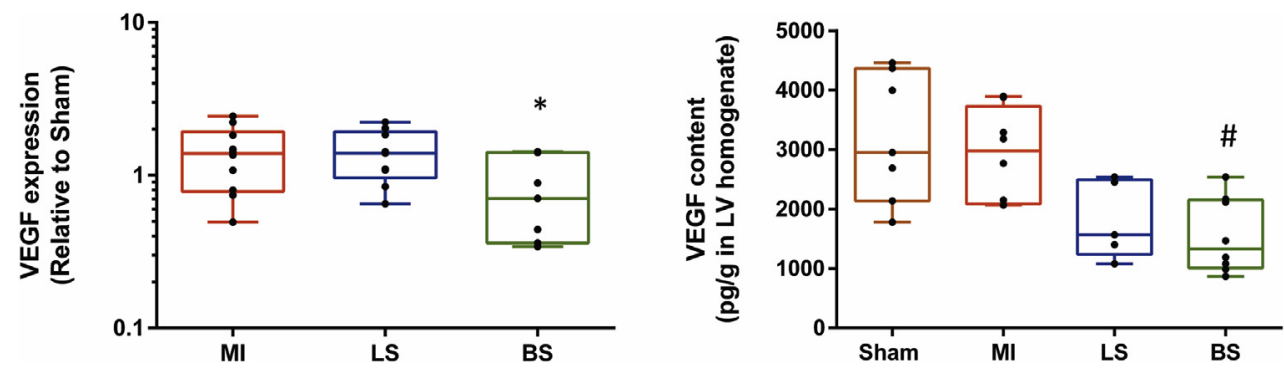

FIGURE 4. Gene expression and protein content of VEGF in the left ventricular myocardium. Data are presented as mean \pm standard deviation for 7 animals in each group. ${ }^{*} P<.01$ versus sham; ${ }^{\#} P<.05$ versus MI. VEGF, Vascular endothelial growth factor; $M I$, myocardial infarction; $L S$, left sympathectomy; $B S$, bilateral sympathectomy. 
Bcl-2

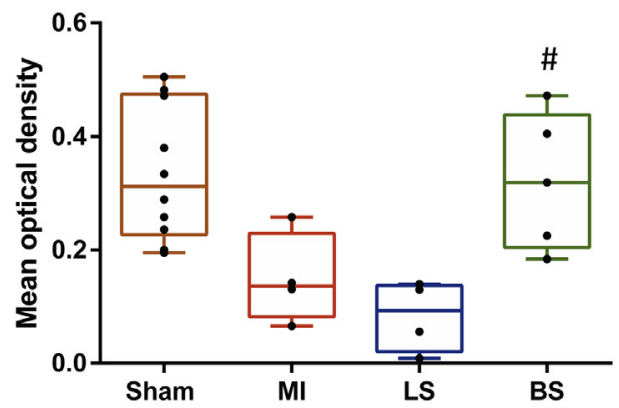

Caspase-3

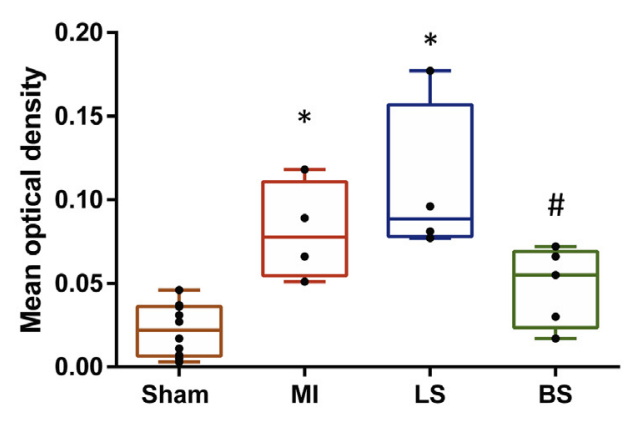

FIGURE 5. Bcl-2 and caspase-3 expression in the left ventricular myocardium. Data are presented as mean \pm standard deviation for 7 animals in each group. ${ }^{*} P<.01$ versus sham; ${ }^{\#} P<.05$ versus MI. $M I$, Myocardial infarction; $L S$, left sympathectomy; $B S$, bilateral sympathectomy.

progression of the inflammatory process will promote hypertrophy and dilatation of the non-infarcted area. ${ }^{14,15}$

The remote postinfarction remodeling of the $\mathrm{LV}$ wall also is associated normally with increased myocardial apoptosis. ${ }^{19}$ Apoptosis is orchestrated by $\mathrm{Bcl}$ and caspase family proteins. Bcl-2 is a family of anti-apoptotic proteins (Bcl-2, Bcl-xL, and $\mathrm{Mcl}-1$ ) that regulates apoptosis in the intrinsic (or mitochondrial) pathway, whereas caspase-3, a pro-apoptotic protein, acts at the cell surface (extrinsic pathway). ${ }^{20}$ Our results demonstrated down-regulation of Bcl-2 after MI was abolished by BS, as well the up-regulation of caspase-3.

The changes in VEGF expression also are relevant in the early phase after MI. The presence of this protein is essential for the neovascularization process, especially at the edges of the myocardial scar, justifying the current research on different methods to promote its supply. ${ }^{21}$ Improvement of cardiac VEGF gene expression also was suggested as an important mechanism underlying the short-term positive effect of sympathetic down-regulation on cardiac function and structure after surgical renal denervation. $^{22}$

In contrast, the current data showed that myocardial gene expressions of MMPs and VEGF were decreased significantly after BS. These changes were associated with the attenuation of LV remodeling and extracellular matrix modifications after MI. The relationship between the activation of cardiac sympathetic drive and the disruption of the myocardial collagen matrix was initially demonstrated by Chen and colleagues, ${ }^{17}$ and its relevance for LV remodeling after MI has been discussed by others. ${ }^{16}$ The modification of metalloproteinases expression is associated with the myocardial remodeling process induced by $\mathrm{MI}^{15}$ and other cardiomyopathies, ${ }^{23,24}$ making its inhibition an important new target for the treatment of chronic heart failure. Similarly, the late increase in VEGF expression also has been associated recently with the fibrogenic response of the myofibroblasts, ${ }^{25,26}$ showing that its long term decrease may be related to the successful inhibition of the extracellular matrix. The interruption of this process also was characterized by the inhibition of myocardial apoptosis in our data, as also observed with cardiac sympathetic afferent denervation after epicardial application of resiniferatoxin. ${ }^{27}$

The interruption of the myocardial remodeling process after MI with BS observed in the current study also resulted in improved LV systolic function. This occurred with the maintenance of a similar LV diastolic volume compared with sham experiments, despite the existence of a significant MI scar. Regardless of the absence of significant modifications of steady-state hemodynamic parameters with BS in relation to the MI group, the functional indexes derived from pressure-volume loop analysis under pharmacologic stress clearly demonstrated its impact on recruitable stroke work and myocardial efficiency.

Thoracic sympathectomy is a safe and less-invasive procedure that may be performed under a video-assisted thoracic surgical approach for either hyperhidrosis ${ }^{28}$ or for the treatment of refractory ventricular arrhythmias. ${ }^{4}$ Several studies documented the absence of important complications related to heart function and exercise performance when this procedure was performed bilaterally for hyperhidrosis treatment. ${ }^{29}$ In patients with heart failure, we demonstrated previously that thoracic sympathectomy can be performed safely, with a positive impact on physical activity. ${ }^{5}$ Furthermore, BS or LS denervation has been investigated recently for the treatment of refractory ventricular arrhythmias. It has significant results and very low complication rates ${ }^{4,30}$ and may prevent arrhythmic disorders after MI. ${ }^{6,31}$ One clinical phase 2 study including patients with New York Heart Association class II and III heart failure and LVEF $<35 \%$ (Neural Cardiac Therapy for Heart Failure; NECTAR-HF) evaluated cardiac sympathetic modulation by vagal nerve stimulator. This study did not demonstrate significant improvements in LVEDV, LVEF, or pro-BNP with vagal nerve stimulator therapy, but patients did report a significant increase in quality of life. $^{32}$

The current preclinical evaluation of the impact of thoracic BS on LV remodeling after MI demonstrated 
that this procedure consistently inhibits the unfavorable development of the myocardial extracellular matrix in the ischemic cardiomyopathy model, limiting the deleterious effects of the activation of cardiac adrenergic drive to support weakening heart function. Beta-blockers and angiotensin-converting enzyme inhibitors are unquestionably the gold standard treatment of post-MI heart failure, and they are known to slow ventricular remodeling progression. Inhibition of collagen formation and detrimental extracellular matrix changes also are obtained with these pharmacologic measures. ${ }^{33,34}$ Nevertheless, an experimental study comparing the efficacy of sympathetic renal denervation and pharmacological therapy in rats after MI showed surprising results. ${ }^{35}$ In addition to improving LVEF and LVEDV, renal denervation alone consistently reduced plasma norepinephrine, renin, angiotensin II, and aldosterone levels, and was thus superior to treatment with beta-blockers or angiotensin-converting enzyme inhibitors.

The current preclinical findings reinforce the use of thoracic BS for control of sympathetic hyperactivity and demonstrate that interruption of sympathetic activity may disrupt different pathways associated with myocardial remodeling and ventricular dysfunction. This positive outcome may be associated with the known positive influence of thoracic sympathectomy on the prevention of refractory ventricular arrhythmias in patients with various cardiac diseases ${ }^{4,6,30}$; thus, this procedure may be an alternative therapeutic option in the management of myocardial remodeling and dysfunction in patients with severe compromise of LV function after MI. Further studies are warranted to investigate the effects of this surgical approach on dilated cardiomyopathy experimental models and patients with chronic heart failure due to various myocardial diseases.

\section{Conflict of Interest Statement}

Authors have nothing to disclose with regard to commercial support.

\section{References}

1. Shen MJ, Zipes DP. Interventional and device-based autonomic modulation in heart failure. Heart Fail Clin. 2015;11:337-48.

2. Singh JP, Kandala J, Camm AJ. Non-pharmacological modulation of the autonomic tone to treat heart failure. Eur Heart J. 2014;35:77-85.

3. Guo W, Liu F, Fu L, Qu R, Wang G, Zhang C. Effects of high thoracic epidural sympathetic blockade for the treatment of severe chronic heart failure due to dilated cardiomyopathy. Acta Cardiol. 2012;67:533-9.

4. Hofferberth SC, Cecchin F, Loberman D, Fynn-Thompson F. Left thoracoscopic sympathectomy for cardiac denervation in patients with life-threatening ventricular arrhythmias. J Thorac Cardiovasc Surg. 2014;147:404-9.

5. Conceição-Souza GE, Pêgo-Fernandes PM, Cruz F, Guimarães GV, Bacal F, Vieira ML, et al. Left cardiac sympathetic denervation for treatment of symptomatic systolic heart failure patients: a pilot study. Eur J Heart Fail. 2012;14:1366-73.
6. De Ferrari GM, Schwartz PJ. Left cardiac sympathetic denervation in patients with heart failure: a new indication for an old intervention? J Cardiovasc Transl Res. 2014;7:338-46.

7. Schwartz PJ, La Rovere MT, De Ferrari GM, Mann DL. Autonomic modulation for the management of patients with chronic heart failure. Circ Heart Fail. 2015;8:619-28.

8. Wu Y, Yin X, Wijaya C, Huang MH, McConnell BK. Acute myocardial infarction in rats. J Vis Exp. 2011; pii 2464.

9. Gulcu N, Gonca E, Kocoglu H. A lateral percutaneous technique for stellate ganglion blockade in rats. Anesth Analg. 2009;108:1701-4.

10. Van den Bergh A, Flameng W, Herijgers P. Parameters of ventricular contractility in mice: influence of load and sensitivity to changes in inotropic state. Pflugers Arch. 2008;455:987-94.

11. Hu J, Yan Y, Zhou Q, Ji M, Niu C, Hou Y, et al. Effects of renal denervation on the development of post-myocardial infarction heart failure and cardiac autonomic nervous system in rats. Int J Cardiol. 2014;172:e414-6.

12. Li Y, Xuan YH, Liu SS, Dong J, Luo JY, Sun ZJ. Shortterm vagal nerve stimulation improves left ventricular function following chronic heart failure in rats. Mol Med Rep. 2015;12:1709-16.

13. Lizotte-Waniewski M, Brew K, Hennekens CH. Hypothesis: metalloproteinase inhibitors decrease risks of cardiovascular disease. J Cardiovasc Pharmacol Ther. 2016;21:368-71.

14. Anzai T. Post-infarction inflammation and left ventricular remodeling: a double-edged sword. Circ J. 2013;77:580-7.

15. Sutton MG, Sharpe N. Left ventricular remodeling after myocardial infarction: pathophysiology and therapy. Circulation. 2000;101:2981-8.

16. Zheng X, Li X, Lyu Y, He YY, Wan WG, Zhu HL, et al. Renal sympathetic denervation improves left ventricular remodeling through regulating MMP9/TIMP1 balance and TGF- $\beta 1$ following myocardial infarction. Exp Physiol. 2016;101:260-71.

17. Cheng CC, Tung KC, Fu YC, Gong CL, Chen YT, Lin NN, et al. Activated matrix metalloproteinase and disrupted myocardial collagen matrix in increased sympathetic activity following stimulation of dorsal medulla in the vagotomized feline model. Chin J Physiol. 2008;51:7-12.

18. Zornoff LA, Paiva SA, Minicucci MF, Spadaro J. Experimental myocardium infarction in rats: analysis of the model. Arq Bras Cardiol. 2009;93:434-40, 426-32.

19. Yeh CC, Li H, Malhotra D, Turcato S, Nicholas S, Tu R, et al. Distinctive ERK and p38 signaling in remote and infarcted myocardium during post-MI remodeling in the mouse. $J$ Cell Biochem. 2010;109:1185-91.

20. Movassagh M, Foo RS. Simplified apoptotic cascades. Heart Fail Rev. 2008;13: 111-9.

21. Rosano JM, Cheheltani R, Wang B, Vora H, Kiani MF, Crabbe DL. Targeted delivery of VEGF after a myocardial infarction reduces collagen deposition and improves cardiac function. Cardiovasc Eng Technol. 2012;3:237-47.

22. Amann K, Odoni G, Benz K, Campean V, Jacobi J, Hilgers KF, et al. Sympathetic blockade prevents the decrease in cardiac VEGF expression and capillary supply in experimental renal failure. Am J Physiol Renal Physiol. 2011;300:F105-12.

23. Polegato BF, Minicucci MF, Azevedo PS, Carvalho RF, Chiuso-Minicucci F, Pereira EJ, et al. Acute doxorubicin-induced cardiotoxicity is associated with matrix metalloproteinase-2 alterations in rats. Cell Physiol Biochem. 2015;35: 1924-33.

24. Yokoseki O, Yazaki Y, Suzuki J, Imamura H, Takenaka H, Isobe M. Association of matrix metalloproteinase expression and left ventricular function in idiopathic dilated cardiomyopathy. Jpn Circ J. 2000;64:352-7.

25. Gordon O, Gilon D, He Z, May D, Lazarus A, Oppenheim A, et al. Vascular endothelial growth factor-induced neovascularization rescues cardiac function but not adverse remodeling at advanced ischemic heart disease. Arterioscler Thromb Vasc Biol. 2012;32:1642-51.

26. Zhao T, Zhao W, Meng W, Liu C, Chen Y, Bhattacharya SK, et al. Vascular endothelial growth factor-D mediates fibrogenic response in myofibroblasts. Mol Cell Biochem. 2016;413:127-35.

27. Wang HJ, Wang W, Cornish KG, Rozanski GJ, Zucker IH. Cardiac sympathetic afferent denervation attenuates cardiac remodeling and improves cardiovascular dysfunction in rats with heart failure. Hypertension. 2014;64:745-55.

28. Cerfolio RJ, De Campos JR, Bryant AS, Connery CP, Miller DL, DeCamp MM, et al. The Society of Thoracic Surgeons expert consensus for the surgical treatment of hyperhidrosis. Ann Thorac Surg. 2011;91:1642-8.

29. Cruz JM, Fonseca M, Pinto FJ, Oliveira AG, Carvalho LS. Cardiopulmonary effects following endoscopic thoracic sympathectomy for primary hyperhidrosis. Eur J Cardiothorac Surg. 2009;36:491-6.

30. Te Riele AS, Ajijola OA, Shivkumar K, Tandri H. Role of bilateral sympathectomy in the treatment of refractory ventricular arrhythmias in arrhythmogenic 
right ventricular dysplasia/cardiomyopathy. Circ Arrhythm Electrophysiol. 2016; 9:e003713.

31. Chen J, Li M, Yu Y, Wu X, Jiang R, Jin Y, et al. Prevention of ventricular arrhythmia complicating acute myocardial infarction by local cardiac denervation. Int J Cardiol. 2015;184:667-73.

32. Zannad F, De Ferrari GM, Tuinenburg AE, Wright D, Brugada J, Butter C, et al. Chronic vagal stimulation for the treatment of low ejection fraction heart failure: results of the NEural Cardiac TherApy foR Heart Failure (NECTAR-HF) randomized controlled trial. Eur Heart J. 2015;36:425-33.

33. St John Sutton M, Ferrari VA. Prevention of left ventricular remodeling after myocardial infarction. Curr Treat Options Cardiovasc Med. 2002;4:97-108.
34. Zannad F, Radauceanu A. Effect of MR blockade on collagen formation and cardiovascular disease with a specific emphasis on heart failure. Heart Fail Rev. 2005; 10:71-8.

35. Hu J, Li Y, Cheng W, Yang Z, Wang F, Lv P, et al. A comparison of the efficacy of surgical renal denervation and pharmacologic therapies in post-myocardial infarction heart failure. PLoS One. 2014;9:e96996.

Key Words: sympathectomy, ventricular remodeling, extracellular matrix

Readers who found these articles interesting may also like to read the following papers found in recent and future issues of our sister publications, Seminars in Thoracic and Cardiovascular Surgery and Operative Techniques in Thoracic and Cardiovascular Surgery!

\section{Acquired: Evolving Technology and Basic Science}

Creation and Global Deployment of a Mobile, Application-Based Cognitive Simulator for Cardiac Surgical Procedures Zachary E. Brewer. Semin Thoracic Surg 2016: 1-9.

"And” is Better Than “Or” Joshua L. Hermsen. Semin Thoracic Surg 2016: 10-11.

Spinal Cord Collateral Pathways -The Road Not Taken Abe DeAnda Jr. Semin Thoracic Surg 2016: 388-9.

Evaluation of a High-fidelity Simulation Training Program for New Cardiothoracic ICU Nurses Bryan Boling, Melanie Hardin-Pierce, Lynne Jensen, Zaki-Udin Hassan. Semin Thoracic Surg 2016: In press. 
TABLE E1. Cytokines, BNP-32, and catecholamines in LV homogenate

\begin{tabular}{lcccr}
\hline & Sham, $\mathbf{n}=\mathbf{7}$ & MI, $\mathbf{n}=\mathbf{7}$ & LS, $\mathbf{n}=\mathbf{7}$ & BS, $\mathbf{n}=\mathbf{7}$ \\
\hline IL-6, pg/g & $9160 \pm 2726$ & $7614 \pm 2221$ & $5402 \pm 1302$ & $5714 \pm 1515$ \\
IL-10, pg/g & $24,996 \pm 9244$ & $17,593 \pm 4229$ & $14,274 \pm 3317^{*}$ & $14,099 \pm 3612^{*}$ \\
BNP-32, pg/g & $4247 \pm 1648$ & $8965 \pm 10,435$ & $4056 \pm 822$ & $5858 \pm 2623$ \\
Adrenaline, ng/g & $17,451 \pm 2680$ & $21,813 \pm 5653$ & $19,120 \pm 5092$ & $22,654 \pm 6462$ \\
Noradrenaline, pg/g & $16,817 \pm 377$ & $17,150 \pm 166^{*}$ & $17,050 \pm 142$ & $17,008 \pm 156$ \\
\hline
\end{tabular}

Data are presented as mean \pm standard deviation for 7 animals in each group. $B N P$, Brain natriuretic peptide; $L V$, left ventricular; $M I$, myocardial infarction; $L S$, left sympathectomy; $B S$, bilateral sympathectomy; $I L$, interleukin. ${ }^{*} P<.05$ versus sham. 